\section{Cell-cycle-related kinase is involved in human glioblastoma carcinogenesis}

Glioblastoma multiforme (GM) is the most aggressive type of brain tumor. Currently, the prognosis for patients with GM is poor. Many genes have been implicated in the tumorigenesis of GM; however, the identification of genes that control the progression of GM is fundamental to the development of new therapies. $\mathrm{Ng}$ et al. have examined the role of cell-cycle-related kinase (CCRK) in GM carcinogenesis.

By comparing the CCRK expression in glioma tissue samples from 26 patients (19 highgrade and 7 low-grade) with that in 3 control samples, the authors showed that $74 \%$ of the high-grade GM samples had elevated CCRK mRNA expression (1.5-3.7-fold). Silencing of CCRK mRNA expression in vitro led to a marked reduction in the proliferation of highly tumorigenic human glioma U-373 MG and U-87 MG cells in a time-dependent and dosedependent manner. In addition, decreased CCRK mRNA expression in these cell lines led to G1/S-phase cell-cycle arrest and decreased phosphorylation of the CDK2 regulator of the G1/S transition. The role of CCRK in carcinogenesis and growth of GM cells in vivo was examined using a nude mouse xenograft model. Silencing of CCRK expression significantly suppressed in vivo growth of $U-373$ and U-87 MG cells $(P<0.001)$. In addition, CCRK overexpression induced malignant transformation of the nontumorigenic U-138 MG cells both in vivo and in vitro.

This is the first report to demonstrate that CCRK has a key role in GM carcinogenesis. Additional studies are required to identify the molecular mechanisms underlying CCRK-mediated oncogenesis.

Original article $\mathrm{Ng} \mathrm{SSM}$ et al. (2007) Cell cycle-related kinase: a novel candidate oncogene in human glioblastoma. J Natl Cancer Inst 99: 936-948

\section{Computer-aided evaluation can improve discrimination of breast lesions on MRI}

Computer-aided evaluation (CAE) programs are used to aid interpretation of MRI images at many breast screening centers. Automatic kinetic assessment by such programs provides detailed kinetics data for the entire lesion. By contrast, manual placement of a region of interest generates kinetics data for only portions of a given lesion. To determine whether kinetics assessment by CAE improves the discrimination between benign and malignant lesions compared with radiologist interpretation, Williams et al. carried out a retrospective study of 154 lesions from 125 women. Histopathological examination determined that 41 of these lesions were malignant.

At threshold enhancement levels of both $50 \%$ and $100 \%$, the sensitivity of CAE was $93 \%$. Three $(7 \%)$ of the malignant lesions did not demonstrate automated threshold enhancement at either level and were, thus, deemed false-negative lesions. At the $50 \%$ threshold, the positive predictive value of $\mathrm{CAE}$ was not significantly different from that of the initial radiologist interpretation $(27.0 \%$ and $26.6 \%$, respectively); however, at the $100 \%$ threshold, the positive predictive value of CAE increased to $30.4 \%$. In addition, when compared with initial radiologist interpretation, CAE at the $100 \%$ threshold reduced false-positive findings by $23.0 \%(P=0.02)$. The authors conclude that, in comparison with radiologist interpretation alone, CAE can improve the discrimination of benign from malignant lesions on MRI, with the improvement being attributable to the absence of threshold enhancement in benign lesions. They state, however, that CAE should not be used as a substitute for radiologist interpretation.

Original article Williams TC et al. (2007) Breast MR imaging: computer-aided evaluation program for discriminating benign from malignant lesions. Radiology 244: 94-103

\section{Survival benefits of combined- modality treatment in elderly patients with NSCLC}

Combined-modality therapy offers survival benefits over radiotherapy alone for patients with non-small-cell lung cancer (NSCLC). Elderly patients have been under-represented in trials of these treatments, however, and no trial has yet directly compared radiotherapy alone with radiotherapy plus chemotherapy in elderly patients with stage III NSCLC. Elderly patients exposed to aggressive combinedmodality treatment might be vulnerable to high toxicity and poor outcomes. 